\title{
Can trained lay providers perform HIV testing services? A review of national HIV testing policies
}

David E. Flynn ${ }^{1,5^{*}}$, Cheryl Johnson², Anita Sands ${ }^{3}$, Vincent Wong ${ }^{4}$, Carmen Figueroa $^{2}$ and Rachel Baggaley ${ }^{2}$

\begin{abstract}
Background: Only an estimated 54\% of people living with HIV are aware of their status. Despite progress scaling up HIV testing services (HTS), a testing gap remains. Delivery of HTS by lay providers may help close this testing gap, while also increasing uptake and acceptability of HIV testing among key populations and other priority groups.

Methods: 50 National HIV testing policies were collated from WHO country intelligence databases, contacts and testing program websites. Data regarding lay provider use for HTS was extracted and collated. Our search had no geographical or language restrictions. This data was then compared with reported data from the Global AIDS Response Progress Reporting (GARPR) from July 2015.

Results: Forty-two percent of countries permit lay providers to perform HIV testing and 56\% permit lay providers to administer pre-and post-test counseling. Comparative analysis with GARPR found that less than half (46\%) of reported data from countries were consistent with their corresponding national HIV testing policy.

Conclusions: Given the low uptake of lay provider use globally and their proven use in increasing HIV testing, countries should consider revising policies to support lay provider testing using rapid diagnostic tests.
\end{abstract}

Keywords: HIV testing, Lay providers, Community health workers, HIV policy

\section{Background}

Despite achievements and expansion of HIV testing services (HTS) to date, presently only $54 \%$ of people living with HIV are aware of their serostatus [1]. It is estimated that there are approximately 2 million new infections annually, half of which are among key populations who often have poor access to HTS, and when they do test, they test late in their infection [2]. Additional and more focused HTS approaches are critical to reach the United Nations "90-90-90" global targets which aims to diagnose $90 \%$ of all people living with HIV by 2020 , have $90 \%$ of those diagnosed receiving sustained antiretroviral therapy (ART) and aiming to have $90 \%$ of those on ART to be virally suppressed [3]. To reach this testing target of $90 \%$, it is critical to address the existing gaps in service

*Correspondence: deflynn75@gmail.com

5 Bellevue St, Chatswood West, NSW 2067, Australia

Full list of author information is available at the end of the article provision, including health worker shortages and the lack of community-based HTS, which limit the expansion and effectiveness of HTS in many resource-limited settings [4-6].

Testing for HIV infection is a crucial part of HTS. Historically, HIV testing involved lengthy laboratory testing of blood collected by venipuncture for HIV antibodies involving specialist equipment and specialist staff. Rapid diagnostic tests (RDTs) for HIV were introduced in the early 2000's and allow for testing of fingerstick whole blood or oral fluid, with a result established in under an hour. Due to their low cost, reliability, ease of use and speed, they are now widely used as a diagnostic means (within a testing algorithm) by low resource countries. Testing with RDTs (both fingerstick blood and oral fluid rapid tests) is possible with adequately trained lay providers. Trained lay providers have been delivering HTS for decades and in many settings [7] across the Americas [8], Europe [9, 10], sub-Saharan Africa [11-17] and Asia [18]. 
Task-sharing - the rational redistribution of tasks from "higher-level" cadres of health professionals to trained lay provider cadres-can expand HTS more broadly, has been shown to increase uptake of HIV testing $[8,12,19]$, and can facilitate testing in community based and outreach services, especially for key populations [20]. Lay providers have been shown to provide high quality services, provide accurate HIV test results [11, 18], and may also be lower in cost than services performed by other health providers.

Since 2008 the World Health Organization (WHO) has recommended task-sharing clinical services within the HIV continuum of diagnosis, prevention, care and treatment [21]. In particular, WHO recommends that programs increase the scope of work of trained lay providers and introduce task-sharing across professional cadres [22]. This task-sharing involves providing clinical services, counseling and referrals to HIV specialist services. However, in many settings lay provider HTS has not been utilized and suboptimal HTS coverage persists.

Global AIDS Response Progress Reporting (GARPR) (WHO, UNAIDS, UNICEF) is an annual initiative that allows representatives from government health departments to voluntarily report information such as epidemiological statistics as well as current clinical HIV testing practices within their country. This information is based upon the clinical practices within the country. According to data from the 2015 Global AIDS Response Progress Reporting (GARPR) (WHO, UNAIDS, UNICEF), only $52 \%(n=65 / 124)$ of reporting countries said that lay providers were allowed to perform HIV rapid diagnostic tests (RDTs) [23]. With this low reported uptake of lay provider use, there is no data regarding the role of HIV national health policies in lay provider utilisation and as a result these policies could be a key barrier to lay provider delivered HTS.

Within this paper we analyzed national HIV testing policies from a range of countries to determine the role of lay providers in delivering HIV testing (through the use of RDTs) as well as pre and post-test counselling. In doing so, we sought to investigate whether national health policy permitted trained lay providers to perform HTS (specifically RDTs and counselling) and compare this to the GARPR reported data to determine whether the national HIV health policy reflects what happens in HIV healthcare systems.

\section{Methods}

\section{Policy criteria}

We collected national policy documents from WHO country intelligence databases, national HIV testing program websites and comprehensive web-based searching through databases and national health department websites. The search and analysis of national HIV policies took place from November 1, 2014 to December 21, 2014. Our search had no geographical or language restrictions. We selected the most recent HIV national testing policies and, when not available, national policy statements on who can perform HTS. We excluded HTS standards and protocols developed by nongovernmental organizations, donors or other technical agencies.

\section{Policy analysis}

We reviewed the policies in full text, extracting three pieces of information: whether trained lay providers can perform HIV rapid tests using finger stick blood, whether trained lay providers can perform HIV rapid tests using oral fluid and whether trained lay providers can perform pre and post-test counselling (which includes the delivering of results). Six researchers (VW, CB, AS, RB, CJ and $\mathrm{DF})$ developed the data extraction form. One reviewer (DF) extracted data, two other reviewers (RB and CJ) evaluated and approved the extracted data. Disagreements between reviewers (RB, CJ and DF) were resolved through discussion and consensus. Our results were then compared with data reported by countries to GARPR (WHO, UNAIDS, UNICEF) as of July 1, 2015, based on the policy and programmatic indicator that asks whether current HIV testing guidelines recommend that RDTs can be performed by lay providers [24].

\section{Global AIDS response progress reporting (GARPR) data}

The GARPR online reporting tool is a method of collection of clinical data and epidemiological statics from a variety of countries on various indicators of global HIV response. Primarily low- and middle-income countries report this data to WHO, UNAIDS and UNICEF. These indicators include epidemiological HIV data as well as a variety of questions regarding HIV practices. Specifically, there are four primary questions about national HIV testing in the GARPR online reporting tool, of which lay provider testing with RDTs is a sub-question. The data is a reflection of the clinical activities that occur within that country (i.e. a reflection of what occurs 'on the ground'), which should ideally be reflected in national HIV policies, however sometimes this is not the case.

National representatives from reporting countries are responsible for providing this information according to the guidance provided by WHO, UNAIDS and UNICEF [24]. These representatives are designated individuals from governmental health departments or government HIV-specific programs

Data regarding whether lay providers can administer HTS in each country was collected in July 2015 and compared to the same information extracted from their corresponding national HIV testing policies. 
The type of HIV testing allowed (i.e. fingerstick or oral fluid based) to be performed by lay providers was not specified in the question the GARPR data collection tool.

\section{Results}

We identified HIV policies from 61 countries and excluded policies from 11 countries because they did not include sufficient information, were not official documents or were not able to be translated. Thus, we included a total of 50 national testing polices in this review: 25 countries were from the WHO African region, four countries from the WHO American region, nine from the WHO Eastern-Mediterranean region, three from the WHO European region, three from the WHO Southeast Asia region, and six from the WHO Western Pacific region.

\section{Analysis of RDTs (using fingerstick blood) use by lay providers}

Twenty-one of 50 national policies allow lay providers to use RDTs using fingerstick blood and 15 of 50 country policies explicitly prohibit lay providers from performing them. Fourteen of 50 countries do not specify a role for lay providers in HIV testing with fingerstick RDTs (Fig. 1).
Analysis of RDTs (using oral fluid) use by lay providers Nine out of the 50 countries had policies allowing lay providers to use RDTs using oral fluid while ten countries specifically did not permit lay providers to use oral fluid RDTs. The majority of countries $(n=31 / 50)$ did not specify if lay providers could use RDTs using oral fluid or not.

\section{Analysis of fingerstick blood vs oral fluid RDT use by lay providers}

Countries that do not permit lay provider RDT testing (with finger stick blood) either do not allow oral fluid testing as well or do not specify if they can perform oral fluid based RDTs. The number of countries that did not allow lay providers to perform either type of RDT was evenly spread across all geographical areas, with no obvious predisposing factor as to why those countries do not permit lay provider testing. Reasons behind the non-use of lay providers were not addressed within the national policies.

\section{Analysis of pre and post-test counselling by lay providers} Twenty-eight of 50 countries permit lay providers to provide pre- and post-test counseling, 12 of 50 countries prohibit lay providers from performing counseling and

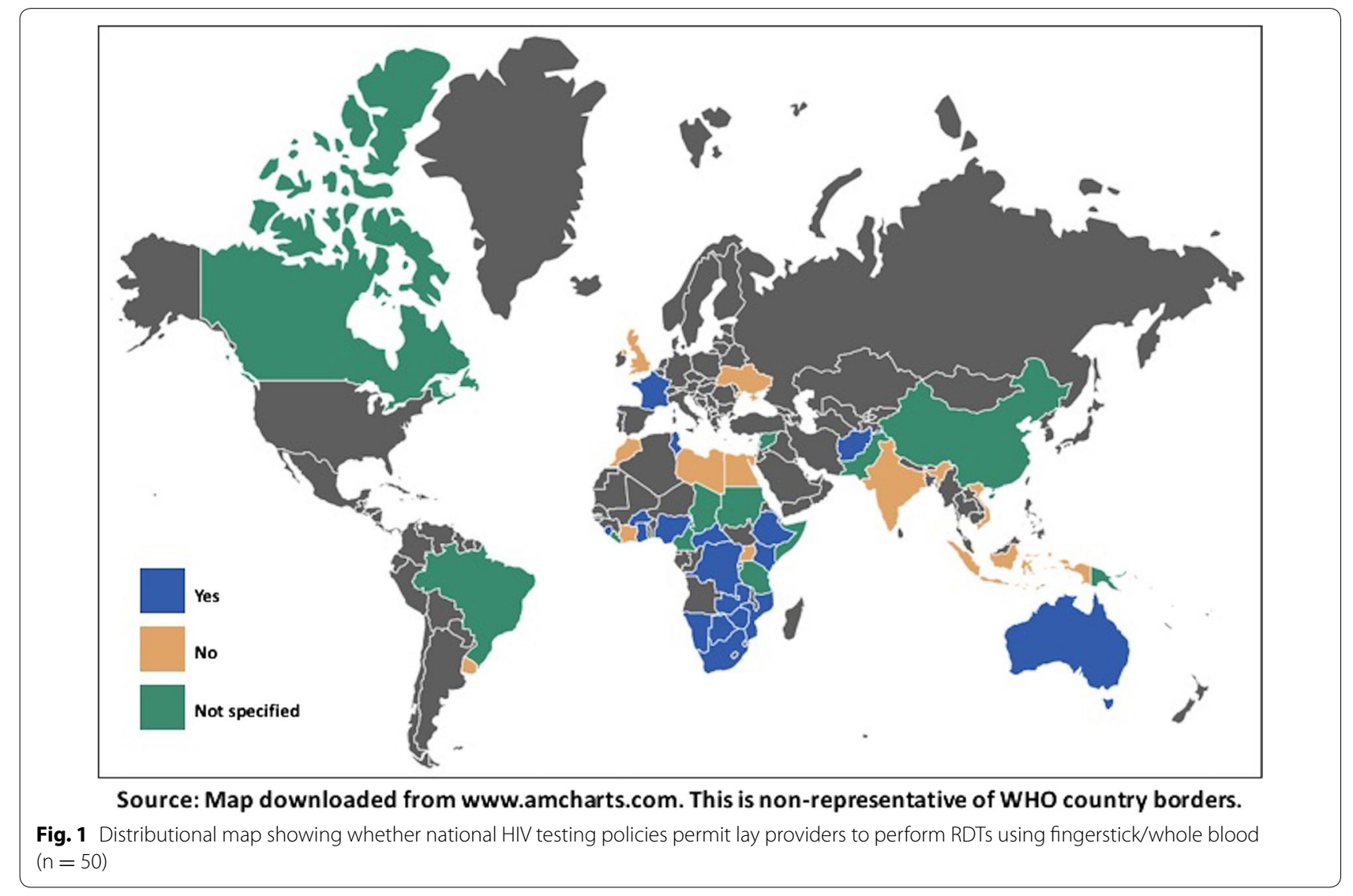




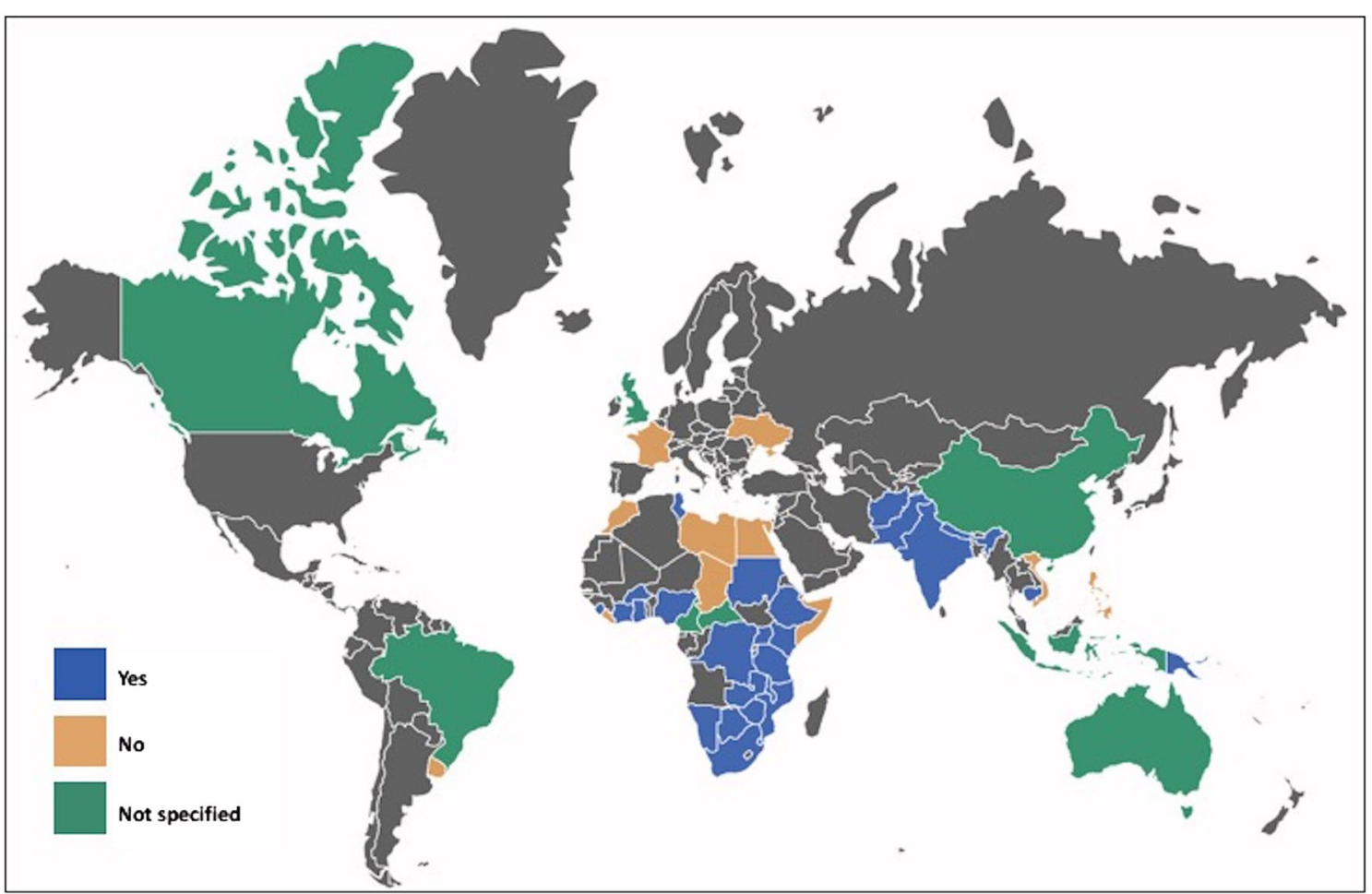

Source: Map downloaded from www.amcharts.com. This is non-representative of WHO country borders.

Fig. 2 Distributional map showing whether national HIV testing policies permit lay providers to perform pre and post-test counseling $(n=50)$

10 of 50 countries did not specify in their policies the role of lay providers in administering counseling (Fig. 2).

\section{Sub-analysis of WHO African region HIV testing policies}

The majority of countries from the WHO African region allow lay providers to perform HIV RDTs using fingerstick/whole blood ( $\mathrm{n}=16 / 25)$ with four countries not allowing lay providers to perform RDTs with fingerstick blood and five countries not specifying the information. Further analysis on the utilization of RDTs using oral fluid shows that only one-fifth $(n=5 / 25)$ of countries in the WHO African region permit lay providers to perform RDTs using oral fluid with three countries not permitting it and 17 of 25 countries not specifying if lay providers can perform RDTs using oral fluid. The WHO African region has the largest percentage of countries per region (80\%) that allow lay providers to perform HIV pre- and post-test counseling $(n=20 / 25)$.

\section{GARPR 2014-2015 data}

A comparison between reviewed national policies and GARPR reporting showed that in 12 of the 50 countries analyzed the national HIV policy and the GARPR data disagreed regarding whether lay providers could perform
HIV testing. Of the 12 policies, five countries reported through GARPR that lay providers cannot perform HTS using RDTs, but their national policy stated that they could. Additionally, seven reviewed policies stated that lay providers were not allowed to provide HTS using RDTs, but according to GARPR reporting they could perform them.

In 23 of 50 countries the HIV testing policies had information that matched the reported GARPR data and 15 of 50 either had HIV testing policies or GARPR data that did not specify the role of lay providers.

GARPR data is not collected on whether lay providers can perform pre and post-test counselling. We were therefore unable to compare our collected data to any reported data.

\section{Raw data}

For further analysis, a table with the raw data of each country is provided as an additional file to this article. It can also be found at: https://figshare.com/s/ a89c08bc4819d5e47db6

The table outlines each country and whether their policy provides lay providers to perform each of the HTS (finger stick blood RDT, oral fluid RDT and pre and post test counselling). 


\section{Discussion}

This is the first review of national policies on HTS for inclusion of lay providers. Similar reviews have been undertaken on HIV policy regarding self-testing [25], but to date there is no information regarding the role of lay providers in national HIV policies.

This review revealed that of the 50 countries analyzed, $58 \%$ either do not permit lay providers to perform HIV RDTs using fingerstick blood (the most common type of HIV RDT) or do not specify if they can, while $44 \%$ do not permit or do not specify whether lay providers can perform HIV pre- and post-test counseling. The regional comparison analysis showed that the WHO African region had the highest proportion of countries in which lay testing is permitted.

Thirteen of the 50 reviewed policies did not specify or explicitly outline the role of lay providers in providing HTS. While some of these countries may have provisions that allow lay providers to perform HIV testing and preand/or post-test counseling, the lack of explicit reference to the role of lay providers may introduce misunderstandings or misinterpretations by providers and national program managers. Furthermore, if lay testing is not explicitly sanctioned, systems to support training, supervision and provide quality assurance of lay testing may not be routinely and consistently applied. Countries should regularly review their national HIV testing policy to make sure it contains correct information, reflects national practice as well as lay provider training, testing and quality assurance.

The difference between reported GARPR data and that extracted from official HIV testing policies may be due to several reasons such as policies that had not been updated to account for lay provider use, incorrect information from the national representative regarding lay provider use in their country, or the policy may not have been clear regarding the role of lay providers in HIV testing.

GARPR data only asks if lay providers are permitted to perform RDTs in general and does not specify whether lay providers are permitted to perform the different types of RDTs (i.e. fingerstick whole blood or oral fluid). This specific information would have helped to give greater clarity on the role of lay providers and aided in our comparison of GARPR data to national HIV policy.

Of the 12 countries with differing information, half $(n=6 / 12)$ of them were from African nations. This difference demonstrates a disparity between reported data and national policy information. Greater care must be taken when reporting GARPR data to make sure it correlates with approved practice. It is suggested that in a bid to increase accuracy, the reporting of GARPR data could be accompanied by policy documents to support their reported data and help catalyze regular reviewing of national policy documents.
Through task-sharing, lay provider HTS have been widely implemented throughout Africa. Sub-analysis of data from the WHO African region suggests that there is a more supportive policy environment for lay provider HTS compared to other global regions. Task sharing to increase the scope of work for lay providers is important to scaling up HTS, as recent reviews have shown that it is acceptable, can be low cost and can increase uptake of HTS, particularly among key populations who are generally underserved [26]. Thus, based on the review of existing evidence, WHO recommends that trained lay providers can perform RDTs safely and effectively [26].

\section{Limitations}

Although we reviewed the most currently available HTS policies, some may be out of date or in the process of being updated. As we collected and analyzed national testing policies, it is possible that some information on lay provider testing was not specified in these, but instead included in national PMTCT guidelines, treatment guidelines or other national guidelines.

Although 50 national policies were collected, this did not match the number of countries reported in GARPR data $(\mathrm{n}=124)$. A greater number of HIV testing policies would have made for a greater comparison and stronger analysis however this difference was explained by the difficulty in obtaining formal HIV policies, the lack of formal HIV policies from a number of the reporting countries and the time limitations that were placed on the analysis.

Data on whether lay providers are permitted to perform pre and post-test counseling was not collected as a part of the 2014-2015 GARPR reporting cycle. Therefore, we could not compare information from reviewed national policies with GARPR reports. Inconsistencies identified between national policies and GARPR reports highlight that the term lay provider may need further definition. It is possible that errors in reporting or missing information may have occurred because of differences in terminology.

\section{Conclusion}

To increase access to and effectiveness of HTS, WHO supports the use of trained lay providers for HIV testing. Trained lay testers can have an important role in providing services in communities to people who do not attend health facilities. Many countries continue to prohibit testing by trained lay testers, and this may be a barrier to reaching those most vulnerable to HIV who do not access existing services.

For countries to increase reach of their HTS, task sharing to lay providers must be a part of a country's testing protocol and should be included in national HIV testing guidelines. Lay provider use is an important way to 
provide acceptable services to key populations, and those other populations who might not present for testing using traditional laboratory-based HTS. Ensuring that RDTs using oral fluid and fingerstick whole blood can be conducted by lay providers will remain the cornerstone of HTS and will be necessary to promote the expansion and uptake of HIV testing.

National representatives must also take greater care when reporting on national HIV testing practices, ensuring that the data reported is up-to-date and reflected in national policies. Regular updating of policies with regards to all aspects of HTC is encouraged.

Increased efforts are needed to accelerate policy adaptation and implementation of the new 2015 WHO Consolidated guidelines on HTS, to help expand HIV testing coverage in areas and among populations with the greatest need.

\section{Additional file}

Additional file 1. Annex 1: Overview of the roles of lay providers according to national HIV testing. Description of Data: A table demonstrating each individual countries use of lay providers in HIV testing for different modalities and HIV pre and post test counselling.

\section{Abbreviations}

HTS: HIV testing services; GARPR: global AIDS response progress reporting; WHO: World Health Organisation; UNAIDS: The Joint United Nations Programme on HIV and AIDS; UNICEF: The United Nations Children's Fund; RDT: rapid diagnostic test.

\section{Authors' contributions}

DF undertook the primary analysis of the national policies, collated the data and aided in a raw draft and final manuscript of the piece. CJ helped to design the study, collate national policies, helped to clarify data and results which were not agreed on by the primary reviewer and helped to write the raw draft of the study with particular emphasis on the literature review. AS was integral in developing the data collection tool as well as the statistical analysis and approving the final draft. VW helped to retrieve national policies and worked on the primary analysis of the policies. CF helped with the statistical analysis of the results, double-checked the results and provided feedback on drafts through the review process. RB conceived the study and coordinated the authors in their various roles. She was instrumental in collecting policies from contacts and reviewed the final set of manuscripts for publication. All authors read and approved the final manuscript.

\section{Authors' information}

DF is a medical student who undertook an unpaid internship at the World Health Organisation to work collaboratively with members of the Key Population and Prevention team within HIV department (working collaboratively with associate Vincent Wong from USAID) to undertake this project.

\section{Author details}

${ }^{1}$ Griffith University School of Medicine, Griffith University, Gold Coast, QLD, Australia. ${ }^{2}$ HIV Department, World Health Organization (WHO), Geneva, Switzerland. ${ }^{3}$ Essential Medicines and Health Products, World Health Organization (WHO), Geneva, Switzerland. ${ }^{4}$ Global Health Bureau: Office of HIV/AIDS, United States Agency for International Development (USAID), Washington, DC, USA. ${ }^{5} 8$ Bellevue St, Chatswood West, NSW 2067, Australia.

\section{Acknowledgements}

Permission was granted by 'amCharts' (www.amcharts.com) for the use and alternation of their world map for the use of Figs. 1 and 2.

\section{Competing Interests}

DEF The author declares that they have no competing interests. CJ Employee of the World Health Organisation (WHO) where this research was undertaken. The WHO will be financing the article processing charge. The organisation does not stand to gain financially or non-financially from this re

search. AS Employee of the World Health Organisation (WHO) where this research was undertaken. The WHO will be financing the article processing charge. The organisation does not stand to gain financially or non-financially from this research. VW The author declares that they have no competing interests. CF Employee of the World Health Organisation (WHO) where this research was undertaken. The WHO will pbe financing the article processing charge. The organisation does not stand to gain financially or non-financially from this research. RB Employee of the World Health Organisation (WHO) where this research was undertaken. The WHO will be financing the article processing charge. The organisation does not stand to gain financially or nonfinancially from this research.

\section{Availability of data and materials}

Raw data is provided as an additional file to this paper, titled: 'Annex 1: Overview of the roles of lay providers according to national HIV testing'. It can also be accessed via figshare at: https://figshare.com/s/a89c08bc4819d5e47db6. GARPR data is not publically available in tabulated form, but is available from the UNADIS website: http://www.unaids.org/AIDSReporting. Alternatively, contact aidsreporting@unaids.org for additional support.

\section{Databases}

All databases within the research paper are publically available with the exception of the World Health Organisation Country (WHO) Intelligence Databases and GARPR Data. Both the WHO Country Intelligence Databases and GARPR data is not publically available. Permission to access both databases/ data was given by Ms Cheryl Johnson (Technical Officer in the Department for HIV at the WHO and co-author of the paper) and overseen by Dr. Rachel Baggaley (Coordinator of HIV Key Population and Prevention unit at the WHO and co-author of this paper).

\section{Ethics approval and consent to participate}

As determined by the WHO Ethics Review Committee, this study did not require ethics approval as it is a policy review and does not contain any human data.

\section{Funding}

DF undertook this project as an unpaid intern and did not receive any grants/ funding for this project. CJ, AS, CF and RB are all employees of the World Health Organisation, while VW is an employee of The United States Agency for International Development (USAID). No grants/external funds were acquired for this research.

Received: 11 February 2016 Accepted: 13 December 2016

Published online: 04 January 2017

\section{References}

1. The Joint United Nations Programme on HIV/AIDS (UNAIDS). How AIDS changed everything -MDG6: 15 years, 15 lessons of hope from the AIDS response. Geneva: Joint United Nations Programme on HIV/AIDS (UNAIDS); 2015

2. The Joint United Nations Programme on HIV/AIDS (UNAIDS). AIDS by the numbers. Geneva: Joint United Nations Programme on HIV/AIDS (UNAIDS); 2013.

3. The Joint United Nations Programme on HIV/AIDS (UNAIDS). 90-90-90: An ambitious treatment target to help end the AIDS epidemic. Geneva: Joint United Nations Programme on HIV/AIDS (UNAIDS); 2014.

4. Samb B, Celletti F, Holloway J, Van Damme W, De Cock K, Dybul M. Rapid expansion of the health workforce in response to the HIV epidemic. $N$ Engl J Med. 2007;357(24):2510-4.

5. Zachariah R, Ford N, Philips M, Lynch S, Massaquoi M, Janssens V, et al. Task shifting in HIV/AIDS: opportunities, challenges and proposed actions for sub-Saharan Africa. Trans R Soc Trop Med Hyg. 2009;103(6):549-58. 
6. Parekh B, Kalou M, Alemnji G, Ou C-Y, Gershy-Damet G-M, Nkengasong J. Scaling up HIV rapid testing in developing countries comprehensive approach for implementing quality assurance. Am J Clin Pathol. 2010;134(4):573-84.

7. Walsh A, Ndubani P, Simbaya J, Dicker P, Brugha R. Task sharing in Zambia: HIV service scale-up compounds the human resource crisis. BMC Health Serv Res. 2010;10(1):272.

8. Walensky R, Reichmann W, Arbelaez C, Wright E, Katz J, Seage I, et al. Counselor-versus provider-based HIV screening in the emergency department: results from the universal screening for HIV Infection in the Emergency Room (USHER) randomized controlled trial. Ann Emerg Med. 2011;58(1):126-32.

9. Champenois K, Le Gall J, Jacquemin C, Jean S, Martin C, Rios L, et al. ANRS-COM'TEST: description of a community-based HIV testing intervention in non-medical settings for men who have sex with men. BMJ Open. 2012;2(2):693

10. Lorente N, Preau M, Vernay-Vaisse C, Mora M, Blanche J, Otis J. Expanding access to non-medicalized community-based rapid testing to men who have sex with men: an urgent HIV prevention intervention (the ANRSDRAG study). PLoS ONE. 2013;8(4):e61225.

11. Molesworth A, Ndhlovu R, Banda E, Ngwira B, Glynn J, Crampin A, et al. High accuracy of home-based community rapid HIV testing in rural Malawi. J Acquir Immune Defic Syndr. 2010;55(5):625-30.

12. Bemelmans M, Van den Akker T, Ford N, Philips M, Zachariah R, Harries A, et al. Providing universal access to antiretroviral therapy in Thyolo, Malawi through task shifting and decentralization of HIV/AIDS care. Trop Med Int Health. 2010;15(12):1413-20.

13. Jackson D, Naik R, Tabana H, Pillay M, Madurai S, Zembe W, et al. Quality of home-based rapid HIV testing by community lay counsellors in a rural district of South Africa. J Int AIDS Soc. 2013;16(1):18744.

14. Fylkesnes K, Sandoy I, Jurgensen M, Chipimo P, Mwangala S, Michelo C. Strong effects of home-based voluntary HIV counselling and testing on acceptance and equity: a cluster randomised trial in Zambia. Soc Sci Med. 2013;86:9-16

15. Iwu E, Holzemer W. Task shifting of HIV management from doctors to nurses in Africa: clinical outcomes and evidence on nurse self-efficacy and job satisfaction. AIDS Care. 2014;26(1):42-52.

16. Jurgensen M, Sandoy I, Michelo C, Fylkesnes K. Effects of home-based Voluntary Counselling and Testing on HIV-related stigma: findings from a cluster-randomized trial in Zambia. Soc Sci Med. 2013;81:18-25.
17. Leon N, Naidoo P, Mathews C, Lewin S, Lombard C. The impact of provider-initiated (opt-out) HIV testing and counseling of patients with sexually transmitted infection in Cape Town, South Africa: a controlled trial. Implement Sci. 2010;5(8):11.

18. Kanal K, Chou T, Sovann L, Morikawa Y, Mukoyama Y, Kakimoto K. Evaluation of the proficiency of trained non-laboratory health staff and laboratory technicians using a rapid and simple HIV antibody test. AIDS Res Ther. 2005;2(1):5.

19. Mathews R, Johnson C, Verster A, Baggaley R. Annex 11: a systematic review of community-based HIV testing and counselling among key populations. In: Consolidated guidelines on HIV testing services: 5Cs: consent, confidentiality, counselling, correct results and connection. Geneva: World Health Organisation; 2015. http://www.ncbi.nlm.nih.gov/books/ NBK316038/.

20. World Health Organisation. Annex 3: global examples of HIV testing servies. In: Consolidated guidelines on HIV testing services. Geneva: World Health Organisation; 2015. http://apps.who.int/iris/bitstream/10665/180212/1/WHO_HIV_2015.22_eng.pdf. Accessed 12 June 2015

21. World Health Organisation. Optimizing health worker roles to improve access to key maternal and newborn health interventions through taskshifting. Geneva: World Health Organization; 2014

22. World Health Organisation. Global recommendations and guidelines on task shifting. Geneva: World Health Organization; 2007. http://www.who. int/workforcealliance/knowledge/resources/taskshifting_guidelines/en/. Accessed June 152015

23. World Health Organisation. Fact sheet to the WHO consolidated guidelines on HIV testing services. Geneva: World Health Organisation; 2015. http://www.who.int/hiv/topics/vct/fact_sheet/en/. Accessed July 17 2015

24. World Health Organisation. Global AIDS response progress reporting 2015. Geneva: World Health Organisation and UNAIDS; 2015.

25. Mavedzenge SN, Baggaley R, Corbett EL. A review of self-testing for HIV: research and policy priorities in a new era of HIV prevention. Clin Infect Dis. 2013;57(1):126-38.

26. World Health Organisation. Consolidated guidelines on HIV testing services. Geneva: World Health Organisation; 2015. http://www.who.int/ hiv/pub/guidelines/hiv-testing-services/en/. Accessed July 152015.

\section{Submit your next manuscript to BioMed Central and we will help you at every step:}

- We accept pre-submission inquiries

- Our selector tool helps you to find the most relevant journal

- We provide round the clock customer support

- Convenient online submission

- Thorough peer review

- Inclusion in PubMed and all major indexing services

- Maximum visibility for your research

Submit your manuscript at www.biomedcentral.com/submit
BioMed Central 